\title{
Partial structural restoring of two-qubit transferred state
}

\author{
A.I.Zenchuk \\ ${ }^{2}$ Institute of Problems of Chemical Physics RAS, Chernogolovka, Moscow reg., 142432, \\ Russia
}

\begin{abstract}
We consider the communication line with two-qubit sender and receiver, the later is embedded into the four-qubit extended receiver. Using the optimizing unitary transformation on the extended receiver we restore the structure of the non-diagonal part of an arbitrary initial sender's state at the remote receiver at certain time instant. Obstacles for restoring the diagonal part are discussed. We represent examples of such structural restoring in a communication line of 42 spin-1/2 particles.

PACS numbers:
\end{abstract}




\section{INTRODUCTION}

The problem of remote state creation originates from the problem of pure state transfer formulated by Bose [1] and becomes an attracting branch in the area of quantum information processing. Apparently, the state initially created at the sender of a communication line can not be transferred to the receiver unless special protocols are implemented. Among the first protocols we refer to that of perfect state transfer [2 44], remote boundary [5, 6] and optimized boundary [7-11] state transfer. Later the remote state creation protocols have been proposed and first realized for the photon systems [12 14], where photons are considered as a basic couriers of quantum information over a long distance. However, the short distance information transfer in quantum information devices can be based on different objects, such as spin chains. As for remote creating a one-qubit state in a spin system, the creatable region in the receiver's state space can be completely described [15, 16] because the one-qubit state-space is parametrized with only three parameters. Thus the one-to-one mapping

$$
\text { initial sender's state } \rightarrow \text { creatable receiver's state }
$$

is established in that case. The complete characterization of creatable region in higher dimensional state-space is much more complicated. Even two-qubit state depends on 15 parameters, so that the mapping (1) can be hardly visualized. Although we can construct certain families of states in this case, like Werner states [17] in Ref.[18], finding the protocol allowing a more careful control of the link between the initial sender's state and the creatable receiver's state is meaningful.

A method of such control is proposed in Ref.[19, 20] where the creation of a two-qubit block-scaled states is considered. In this case the receiver's state defers from the sender's one by the factor ahead of certain blocks of the density matrix. These blocks are multiplequantum (MQ) coherence matrices. Remember that the $n$-order coherence matrix collects those elements of the density matrix which are responsible for the state-transitions changing the $z$-projection of the total spin by $n$ ). The feature of these blocks is that they evolve independently provided that the dynamics is described by the Hamiltonian conserving the $z$-projection of the total spin momentum. However, the protocol proposed in that paper requires a special initial state and is not applicable to an arbitrary one. As a result, each MQ-coherence matrix caries at most one arbitrary parameter. 
In this paper we modify the mentioned protocol by implementing the extended receiver (the subsystem at the receiver side embedding the receiver itself [21]) and the fixed optimizing unitary transformation on it. We emphasize that, being fixed, the optimizing unitary transformation represents a part of the protocol and remains the same for any transferred state. As a result we manage to structurally reconstruct the non-diagonal part of the initial sender's density matrix in the receiver's density matrix at certain time instant, i.e., the nondiagonal elements of the receiver's density matrix become proportional to the appropriate elements of the sender's initial density matrix in our protocol. We also discuss the obstacle arising in restoring the diagonal elements of the initial sender's density matrix.

Let us mention another aspect of our protocol. Apparently, if there is no optimizing unitary transformation, then the elements of the sender's initial density matrix appear in the receiver's density matrix as linear combinations. Thus, the problem of state transfer reduces to the system of linear algebraic equations for the elements of the sender's density matrix which is solvable in general [22]. However, the quantum-mechanical solution of this system in spin-1/2 communication line is not proposed. Implementing the optimizing unitary transformation, we solve the nondiagonal part of this system and find scaled nondiagonal elements of the sender's initial density matrix. Therefore our protocol contributes into the problem of solving the linear algebraic equations via quantum-mechanical methods [23].

The paper is organized as follows. The proposed model of a communication line is described in Sec.II. The general protocol of structural restoring of a two-qubit state, including the optimization of the time instant for state registration and construction of the optimal unitary transformation on the four-qubit extended receiver, is proposed in Sec.III. Examples of numerical structural restoring of non-diagonal elements of a two-qubit initial sender's state in the communication line of $N=42$ nodes are represented in Sec IV General conclusions are given in Sec:D.

\section{MODEL}

We consider the communication line consisting of two-qubit sender $S$ and two-qubit receiver $R$ embedded into the four-node extended receiver $E R$, which is connected to the sender through the transmission line TL, see Fig.1. The spin dynamics is governed by the 


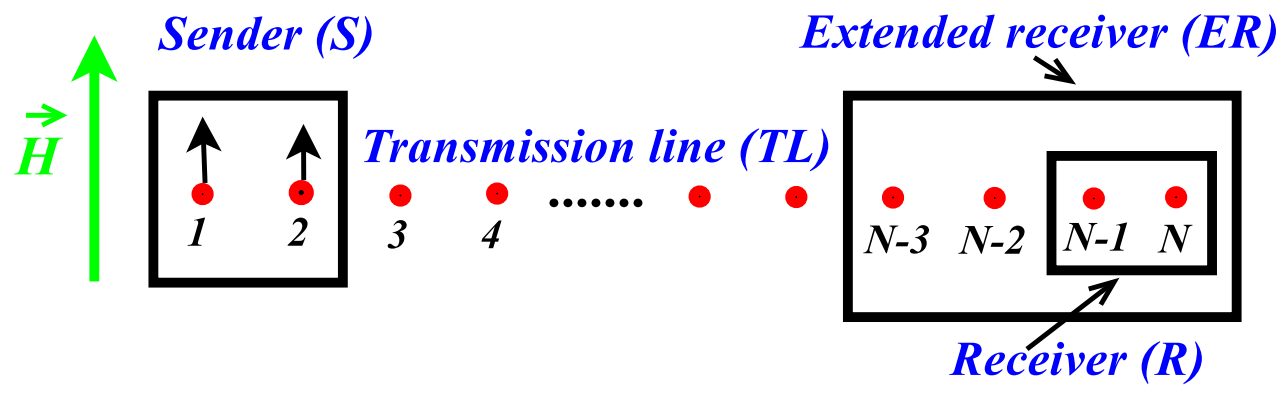

FIG. 1: Communication line including the sender (S), transmission line (TL), receiver (R) and extended receiver (ER). $\vec{H}$ is the external magnetic field.

XX-Hamiltonian with the dipole-dipole interaction

$$
\begin{aligned}
& H=\sum_{j>i} D_{i j}\left(I_{i x} I_{j x}+I_{i y} I_{j y}\right), \\
& {\left[H, I_{z}\right]=0,}
\end{aligned}
$$

where $D_{i j}=\frac{\gamma^{2} \hbar}{r_{i j}^{3}}$ is the coupling constant between the $i$ th and $j$ th nodes, $\gamma$ is the gyromagnetic ratio, $r_{i j}$ is the distance between the $i$ th and $j$ th nodes, $I_{i \alpha}(\alpha=x, y, z)$ is the projection operator of the $i$ th spin on the $\alpha$ axis and $I_{z}=\sum_{i} I_{i z}$. We also consider the tensor-product initial state

$$
\rho(0)=\rho^{(S)}(0) \otimes \rho^{(T L)} \otimes \rho^{(E R)}(0)
$$

where $\rho^{(S)}(0)$ is an arbitrary initial state of the sender $S, \rho^{(T L)}$ and $\rho^{(E R)}$ are the ground states (states without excitations) of the transmission line and extended receiver respectively:

$$
\rho^{(T L)}=\operatorname{diag}(1,0, \ldots), \quad \rho^{(E R)}=\operatorname{diag}(1,0, \ldots) .
$$

Then

$$
\rho^{(R)}(t)=\operatorname{Tr}_{r e s t}\left(\tilde{V}(t) \rho(0) \tilde{V}^{+}(t)\right), \quad \tilde{V}(t)=e^{-i H t} .
$$

Here the trace is over all the nodes of the communication line except the receiver.

In our protocol we use the expansion of the density matrices in the sums of the multiplequantum (MQ) coherence matrices [24] which read in the two-qubit case as

$$
\rho^{(S)}=\sum_{k=-2}^{2} \rho^{(S ; k)}, \quad \rho^{(R)}=\sum_{k=-2}^{2} \rho^{(R ; k)},
$$


where the MQ coherence matrices $\rho^{(S ; k)}$ collect terms responsible for the state transfers changing the $z$-projection of the total spin momentum by $k$.

We emphasize that the commutation condition (3) together with the initial condition (15) (where the initial density matrices $\rho^{(T L)}$ and $\rho^{(E R)}$ include only the zero-order coherence matrix) provides transferring the MQ-coherence matrices from the sender to the receiver without mutual interaction [19].

\section{STRUCTURAL RESTORING OF TRANSFERRED TWO-QUBIT STATE}

Hereafter we use the Dirac notation for the basis in the state-space of $M$-qubit (sub)system:

$$
\left|n_{1} \ldots n_{M}\right\rangle, \quad n_{i}=0,1, \quad i=1, \ldots, M
$$

and the appropriate multi-index $J_{M}=\left\{n_{1} \ldots n_{M}\right\}$. For instance, the elements of a density matrix $\rho$ in the $M$-qubit state-space read

$$
\rho_{n_{1} \ldots n_{M} ; m_{1} \ldots m_{M}}=\left\langle n_{1} \ldots n_{M}|\rho| m_{1} \ldots m_{M}\right\rangle \text {. }
$$

We say that the initial sender's density matrix $\rho^{(S)}(0)$ is structurally restored at the receiver if the elements of the receiver's density matrix are proportional to the corresponding elements of the initial sender's density matrix up to the normalization condition [19]. For the two-qubit state restoring, by virtue of expansions (7) for the sender's and receiver's density matrices, the restored state has the following form:

$$
\begin{aligned}
& \rho_{n_{1} n_{2} ; m_{1} m_{2}}^{(R ;)}=\lambda_{n_{1} n_{2} ; m_{1} m_{2}}^{(k)} \rho_{n_{1} n_{2} ; m_{1} m_{2}}^{(S ; k)}, \quad k= \pm 1, \pm 2, \quad m_{1}+m_{2}-n_{1}-n_{2}=k, \\
& \rho_{n_{1} n_{2} ; m_{1} m_{2}}^{(R ; 0)}=\lambda_{n_{1} n_{2} ; m_{1} m_{2}}^{(0)} \rho_{n_{1} n_{2} ; m_{1} m_{2}}^{(S ; ;)}, m_{1}+m_{2}-n_{1}-n_{2}=0, \\
& \rho_{00 ; 00}^{(R ; 0)}=1-\sum_{k_{1}+k_{2} \neq 0} \lambda_{k_{1} k_{2} ; k_{1} k_{2}}^{(0)} \rho_{k_{1} k_{2} ; k_{1} k_{2}}^{(S ; 0)}, \\
& \lambda_{m_{1} m_{2} ; n_{1} n_{2}}^{(-k)}=\left(\lambda_{n_{1} n_{2} ; m_{1} m_{2}}^{(-k)}\right)^{*}, \quad k=0,1,2, \quad \operatorname{Im} \lambda_{n_{1} n_{2} ; n_{1} n_{2}}^{(0)}=0,
\end{aligned}
$$

where normalization condition (12) provides $\operatorname{Tr} \rho^{(R)}=1$.

\section{A. Time instant for state registration}

To proceed with, we have to find the optimal time instant for state registration at the receiver. Since the second-order coherence intensity is, generally, the smallest one in the 
two-qubit system, we select the time instant corresponding to the maximal value of this quantity. Unlike the other coherences, the second-order coherence includes only one element $\rho_{00 ; 11}^{(R)} \equiv \rho_{00 ; 11}^{(R ; 2)}$ of the density matrix and therefore there is no mixing of elements in this matrix block. For this single element we have

$$
\begin{aligned}
& \rho_{00 ; 11}^{(R)}=\left\langle 00\left|\rho^{(R)}(t)\right| 11\right\rangle= \\
& \sum_{J_{N-2}} \tilde{V}_{J_{N-2} 00 ; 0_{N}} \rho^{(S)}(0)_{00 ; 11} \rho_{0_{N-6} ; 0_{N-6}}^{(T L)} \rho_{0_{4} ; 0_{4}}^{(E R)} \tilde{V}_{110_{N-2} ; J_{N-2} 11}^{+}=\rho_{00 ; 11}^{(S)} \tilde{V}_{0_{N} ; 0_{N}} \tilde{V}_{110_{N-2} ; 0_{N-2} 11}^{+} .
\end{aligned}
$$

Here $0_{K}$ is the set of $K$ zeros. We also use the fact that our system evolves in the twoexcitation subspace due to commutation (3) and initial state (44), (5). Therefore, the sum over $J_{N-2}$ reduces to the single term with $J_{N-2}=0_{N-2}$. Since $\tilde{V}_{0_{N} ; 0_{N}}=1$ (we set the energy of the ground state to be zero, therefore this state does not evolve) we have

$$
\rho_{00 ; 11}^{(R)}=\rho_{00 ; 11}^{(S)} \tilde{V}_{110_{N-2} ; 0_{N-2} 11}^{+}
$$

Consequently, to maximize the second-order coherence intensity $I^{(2)}=\left|\rho_{00 ; 11}^{(R)}\right|^{2}$ we have to maximize $\left|\tilde{V}_{110_{N-2} ; 0_{N-2} 11}\right|^{2}$ which is a smooth function of $t$ with the maximum

$$
\left|\tilde{V}_{110_{N-2} ; 0_{N-2} 11}\right|_{\max }^{2}=0.0151 \text { at } t_{\max }=46.0245
$$

for the chain of $N=42$ nodes. To obtain the larger factor $\left|\tilde{V}_{110_{N-2 ;} 0_{N-2} 11}\right|_{\max }^{2}$ we optimize two boundary pairs of the coupling constants in the spin chain [10]. For convenience, we introduce notation

$$
D_{i(i+1)} \equiv \delta_{i}, \quad i=1, \ldots, N-1
$$

For the homogeneous chain we have $\delta_{i}=\delta, i=1, \ldots, N-1$. For the chain with optimized boundary coupling constants we set

$$
\begin{array}{r}
\delta_{k}=\delta, \quad 3 \leq k \leq N-3, \\
\delta_{1}=\delta_{N-1}, \quad \delta_{2}=\delta_{N-2},
\end{array}
$$

see Fig,2, The maximization of $\left|\tilde{V}_{110_{N-2} ; 0_{N-2} 11}\right|^{2}$ yields

$$
\delta_{1}=0.3005 \delta, \quad \delta_{2}=0.5311 \delta, \quad\left|\tilde{V}_{110_{N-2} ; 0_{N-2} 11}\right|_{\max }^{2}=0.4372, \quad t_{\max }=58.9826
$$

for $N=42$, i.e., $\left|\tilde{V}_{110_{N-2} ; 0_{N-2} 11}\right|_{\text {max }}^{2}$ is increased by the factor of about 29 in comparison with the homogeneous chain. This optimized boundary chain is used below. 


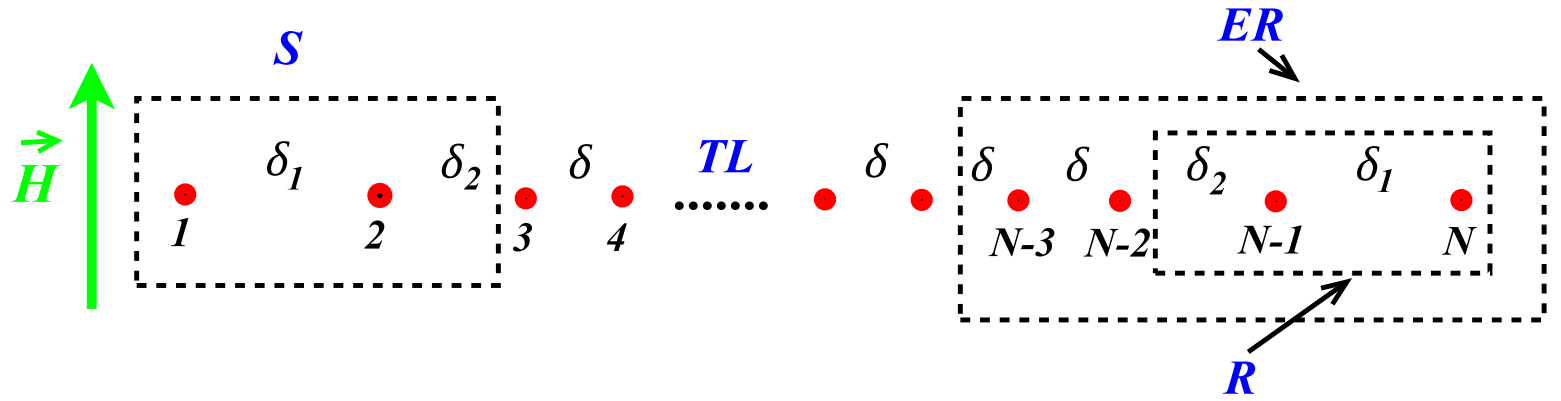

FIG. 2: Communication line shown in Fig 1 with optimized two pairs of boundary coupling constants $D_{1,2}=D_{N-1, N}=\delta_{1}$ and $D_{2,3}=D_{N-2, N-1}=\delta_{2}$.

\section{B. Optimizing unitary transformation}

For restoring the structure of the matrices $\rho^{(S ; k)}$ at the receiver we apply the optimizing local unitary transformation $V$ to the extended receiver, consisting of four nodes in our case. Thus, the total evolution operator $W$ reads

$$
W=V \tilde{V}, \quad V=I_{2^{N-4}} \otimes V_{0} .
$$

To avoid mixing the MQ-coherence matrices, the operator $V_{0}$ has to preserve the $z$-projection of the total spin momentum, i.e.,

$$
\left[V_{0}, I_{z}\right]=0
$$

To better characterize the structure of the unitary transformation, we pass to the scalar indexes from the Dirac notations (8) through the rule

\begin{tabular}{|c|c|c|c|c|c|c|c|c|c|c|c|}
\hline$\left(n_{1} n_{2} n_{3} n_{4}\right)$ & $(0000)$ & $(0001)$ & $(0010)$ & $(0011)$ & $(0100)$ & $(0101)$ & $(0110)$ & $(1000)$ & $(1001)$ & $(1010)$ & $(1100)$ \\
$i$ & 1 & 2 & 3 & 4 & 5 & 6 & 7 & 8 & 9 & 10 & 11 \\
\hline
\end{tabular}

and write the basis of the Lie algebra associated with this unitary transformation. This basis consists of 42 non-diagonal elements $\gamma^{(1 ; i j)}, \gamma^{(2 ; i j)}, j>i$ (diagonal elements are not useful in our transformations) with the following non-zero elements:

$$
\begin{aligned}
& \gamma_{i j}^{(1 ; i j)}=\gamma_{j i}^{(1 ; i j)}=1, \quad \gamma_{i j}^{(2 ; i j)}=-\gamma_{j i}^{(1 ; i j)}=-i \\
(i, j) \in & \{(2,3),(2,5),(2,8),(3,5),(3,8),(4,6),(4,7),(4,9),(4,10),(4,11),(5,8), \\
& (6,7),(6,9),(6,10),(6,11),(7,9),(7,10),(7,11),(9,10),(9,11),(10,11)\} .
\end{aligned}
$$


We represent the restoring operator $V_{0}$ in the form

$$
V_{0}=\prod_{i=1}^{11} \prod_{j>i} e^{i \varphi_{i, j}^{(2)} \gamma^{(2 ; i j)}} e^{i \varphi_{i, j}^{(1)} \gamma^{(1 ; i j)}}
$$

where $\varphi_{i, j}^{(k)}$ are scalar parameters and the product is ordered in such a way that $i$ and $j$ increase from the right to the left.

\section{Restored second-order coherence matrix}

The second-order coherence matrix doesn't require structural restoring because it consists of one element and therefore can be written in form (10):

$$
\begin{aligned}
& \rho_{00 ; 11}^{(R)}=W_{0_{N-2} 00 ; 000_{N-2}} \rho_{00 ; 11}^{(S)} W_{110_{N-2} ; 0_{N-2} 11}^{+}=\lambda_{00 ; 11}^{(2)} \rho_{00 ; 11}^{(S)}, \\
& \lambda_{00 ; 11}^{(2)}=W_{110_{N-2} ; 0_{N-2} 11}^{+} .
\end{aligned}
$$

Here and below we set $W_{0_{N-2} 00 ; 000_{N-2}}=1$ because the ground state does not evolve.

\section{Structural restoring of first-order coherence matrix}

The elements of the first-order coherence matrix after evolution and optimizing transformation read:

$$
\begin{aligned}
\rho_{00 ; n_{1} n_{2}}^{(R)}= & \sum_{i_{1}+i_{2}=1} \rho_{00 ; i_{1} i_{2}}^{(S)} W_{i_{1} i_{2} 0_{N-2} ; 0_{N-2} n_{1} n_{2}}^{+}+ \\
& \sum_{\left|J_{N-2}\right|=1} \sum_{i_{1}+i_{2}=1} W_{J_{N-2} 00 ; i_{1} i_{2} 0_{N-2}} \rho_{i_{1} i_{2} ; 11} W_{110_{N-2} ; J_{N-2} n_{1} n_{2}}^{+} \\
\rho_{n_{1} n_{2} ; 11}^{(R)}= & \sum_{i_{1}+i_{2}=1} W_{0_{N-2} n_{1} n_{2} ; i_{1} i_{2} 0_{N-2}} \rho_{i_{1} i_{2} ; 11}^{(S)} W_{110_{N-2} ; 0_{N-2} 11}^{+},
\end{aligned}
$$

where $\left|J_{N-2}\right|$ means the sum of the elements of the vector index $J_{N-2}$ and $n_{1}+n_{2}=1$. Deriving these equations we take into account the relation

$$
W_{110_{N-2} ; J_{N-2} 11}^{+}=W_{J_{N-2} 11 ; 110_{N-2}}=0 \text { if }\left|J_{N-2}\right| \neq 0
$$

which is a consequence of commutation relations (3) and (20) (transitions changing the $z$-projection of the total spin momentum are forbidden). 
The structural restoring of elements $\rho_{00 ; n_{1} n_{2}}^{(S)}$ and $\rho_{n_{1} n_{2} ; 11}^{(S)}$ (described by eqs.(27) and (28) $)$ requires, respectively,

$$
\begin{aligned}
& \sum_{\left|J_{N-2}\right|=1} W_{J_{N-2} 00 ; m_{1} m_{2} 0_{N-2}} W_{110_{N-2} ; J_{N-2} n_{1} n_{2}}^{+}=0, \quad n_{1}+n_{2}=m_{1}+m_{2}=1 \\
& W_{n_{1} n_{2} 0_{N-2} ; 0_{N-2} n_{2} n_{1}}^{+}=0 \Leftrightarrow W_{0_{N-2} n_{2} n_{1} ; n_{1} n_{2} 0_{N-2}}=0, \quad n_{1}+n_{2}=1 .
\end{aligned}
$$

Then equations (27) and (28) get the structurally restored form (10):

$$
\begin{aligned}
& \rho_{00 ; n_{1} n_{2}}^{(R)}=\lambda_{00 ; n_{1} n_{2}}^{(1)} \rho_{00 ; n_{1} n_{2}}^{(S)}, \\
& \rho_{n_{1} n_{2} ; 11}^{(R)}=\lambda_{n_{1} n_{2} ; 11}^{(1)} \rho_{n_{1} n_{2} ; 11}^{(S)},
\end{aligned}
$$

where $n_{1}+n_{2}=1$ and

$$
\lambda_{00 ; n_{1} n_{2}}^{(1)}=W_{n_{1} n_{2} 0_{N-2} ; 0_{N-2} n_{1} n_{2}}^{+}, \quad \lambda_{n_{1} n_{2} ; 11}^{(1)}=W_{0_{N-2} n_{1} n_{2} ; n_{1} n_{2} 0_{N-2}} W_{110_{N-2} ; 0_{N-2} 11}^{+} .
$$

Thus, all in all, we have to solve system (30), (31) of 6 complex equations for the $\varphi$ parameters of the unitary transformation $V_{0}$. This system is equivalent to the 12 real-valued equations which can be solved using at least $12 \phi$-parameters of 42-parametric unitary transformation (24).

\section{E. Structural restoring of zero-order coherence matrix}

The elements of the zero-order coherence matrix after the optimizing unitary transformation read:

$$
\begin{aligned}
& \rho_{n_{1} n_{2} ; m_{1} m_{2}}^{(R ; 0)}=\sum_{\left|J_{N-2}\right|=1} W_{J_{N-2} n_{1} n_{2} ; 110_{N-2}} \rho_{11 ; 11}^{(S ; 0)} W_{110_{N-2} ; J_{N-2} m_{1} m_{2}}^{+}+ \\
& \sum_{\substack{i_{1}+i_{2}=1 \\
j_{1}+j_{2}=1}} W_{0_{N-2} n_{1} n_{2} ; i_{1} i_{2} 0_{N-2}} \rho_{i_{1} i_{2} ; j_{1} j_{2}}^{(S ; 0)} W_{j_{1} j_{2} 0_{N-2} ; 0_{N-2} m_{1} m_{2}}^{+}, n_{1}+n_{2}=m_{1}+m_{2}=1, \\
& \rho_{11 ; 11}^{(R ; 0)}=\lambda_{11 ; 11}^{(0)} \rho_{11 ; 11}^{(S ; 0)}, \quad \lambda_{11 ; 11}^{(0)}=W_{0_{N-2} 11 ; 110_{N-2}} W_{110_{N-2} ; 0_{N-2} 11}^{+}, \\
& \rho_{00 ; 00}^{(R ; 0)}=\rho_{00 ; 00}+\sum_{\left|J_{N-2}\right|=1} \sum_{\substack{i_{1}+i_{2}=1 \\
j_{1}+j_{2}=1 \\
(S ; 0)}} W_{J_{N-2} 00 ; i_{1} i_{2} 0_{N-2}} \rho_{i_{1} i_{2} ; j_{1} j_{2}}^{(S ; 0)} W_{j_{1} j_{2} 0_{N-2} ; J_{N-2} 00}^{+}+ \\
& \sum_{\left|J_{N-2}\right|=2} W_{J_{N-2} 00 ; 110_{N-2}} \rho_{11 ; 11}^{S ; 1} W_{110_{N-2} ; J_{N-2} 00}^{+} .
\end{aligned}
$$

These equations can be simplified in the case of structural restoring of the first order coherence matrix. In fact, first, since $W$ is a unitary transformation, we have

$$
\sum_{\left|J_{N-2}\right|=1} W_{J_{N} ; n_{1} n_{2} 0_{N-2}} W_{n_{2} n_{1} 0_{N-2} ; J_{N}}^{+}=0 .
$$


Furthermore, in view of restoring conditions (31), eq.(37) reduces to the form

$$
\sum_{\left|J_{N-2}\right|=1} W_{J_{N-2} 00 ; n_{1} n_{2} 0_{N-2}} W_{n_{2} n_{1} 0_{N-2} ; J_{N-2} 00}^{+}=0 .
$$

Finally, using eqs.(31) and (38), respectively, in eqs. (34) and (36) we write them as

$$
\begin{aligned}
& \rho_{n_{1} n_{2} ; m_{1} m_{2}}^{(R ; 0)}=\sum_{\left|J_{N-2}\right|=1} W_{J_{N-2} n_{1} n_{2} ; 110_{N-2}} \rho_{11 ; 11}^{(S ; 0)} W_{110_{N-2} ; J_{N-2} m_{1} m_{2}}^{+}+ \\
& W_{0_{N-2} n_{1} n_{2} ; n_{1} n_{2} 0_{N-2}} \rho_{n_{1} n_{2} ; m_{1} m_{2}}^{(S ; 0)} W_{m_{1} m_{2} 0_{N-2} ; 0_{N-2} m_{1} m_{2}}^{+}, \quad n_{1}+n_{2}=m_{1}+m_{2}=1, \\
& \rho_{00 ; 00}^{(R ; 0)}=\rho_{00 ; 00}^{(S ; 0)}+\sum_{i_{1}+i_{2}=1} \sum_{\left|J_{N-2}\right|=1}\left|W_{J_{N-2} 00 ; i_{1} i_{2} 0_{N-2}}\right|^{2} \rho_{i_{1} i_{2} ; i_{1} i_{2}}^{(S ; 0)}+ \\
& \sum_{\left|J_{N-2}\right|=2}\left|W_{J_{N-2} 00 ; 110_{N-2}}\right|^{2} \rho_{11 ; 11}^{(S ; 0)} .
\end{aligned}
$$

Eq.(40) is normalization trace-condition (12) and therefore does not require restoring. While restoring of eq.(39) implies the following constraints for the operator $W$ :

$$
\sum_{\left|J_{N-2}\right|=1} W_{J_{N-2} n_{1} n_{2} ; 110_{N-2}} W_{110_{N-2} ; J_{N-1} m_{1} m_{2}}^{+}=0, \quad n_{1}+n_{2}=m_{1}+m_{2}=1 .
$$

System (41) combines two different cases $\left(m_{1}, m_{2}\right)=\left(n_{1}, n_{2}\right)$ and $\left(m_{1}, m_{2}\right)=\left(n_{2}, n_{1}\right)$. Writing these two cases separately $\left(n_{1}+n_{2}=1\right)$,

$$
\begin{aligned}
& \sum_{\left|J_{N-2}\right|=1} W_{J_{N-2} n_{1} n_{2} ; 110_{N-2}} W_{110_{N-2} ; J_{N-1} n_{2} n_{1}}^{+}=0 \\
& \sum_{\left|J_{N-2}\right|=1}\left|W_{J_{N-2} n_{1} n_{2} ; 110_{N-2}}\right|^{2}=0
\end{aligned}
$$

we see that eq. (43) is a sum of positive terms. Therefore it is equivalent to the following system

$$
W_{J_{N-2} n_{1} n_{2} ; 110_{N-2}}=0, \quad n_{1}+n_{2}=1, \quad\left|J_{N-2}\right|=1
$$

Moreover, if eqs.(44) are satisfied, then eqs.(30) and (42) become identities. All in all, the conditions for the complete structural restoring of both the first- and zero-order coherence matrices read $\left(n_{1}+n_{2}=1\right)$

$$
\begin{aligned}
& W_{0_{N-2} n_{2} n_{1} ; n_{1} n_{2} 0_{N-2}}=0, \\
& W_{J_{N-2} n_{1} n_{2} ; 110_{N-2}}=0, \quad\left|J_{N-2}\right|=1 .
\end{aligned}
$$


As a result, eqs. (40) and (39) get the structurally restored forms (11) and (12):

$$
\begin{aligned}
& \rho_{n_{1} n_{2} ; n_{1} n_{2}}^{(R ; 0)}=\lambda_{n_{1} n_{2} ; n_{1} n_{2}}^{(0)} \rho_{n_{1} n_{2} ; n_{1} n_{2}}^{(S ; 0)}, \quad n_{1}+n_{2}=1, \\
& \rho_{n_{1} n_{2} ; n_{2} n_{1}}^{(R ; 0)}=\lambda_{n_{1} n_{2} ; n_{2} n_{1}}^{(0)} \rho_{n_{1} n_{2} ; n_{2} n_{1}}^{(S ; 0)}, \quad n_{1}+n_{2}=1, \\
& \rho_{11 ; 11}^{(R ; 0)}=\lambda_{11 ; 11}^{(0)} \rho_{11 ; 11}^{(S ; 0)}, \\
& \rho_{00 ; 00}^{(R ; 0)}=\rho_{00 ; 00}^{(S ; 0)}-\sum_{i_{1}+i_{2}=1} \lambda_{i_{1} i_{2} ; i_{1} i_{2}}^{(0)} \rho_{i_{1} i_{2} ; i_{1} i_{2}}^{(S ; 0)}-\lambda_{11 ; 11}^{(0)} \rho_{11 ; 11}^{(S ; 0)}
\end{aligned}
$$

where

$$
\begin{aligned}
& \lambda_{n_{1} n_{2} ; n_{2} n_{1}}^{(0)}=W_{0_{N-2} n_{1} n_{2} ; n_{1} n_{2} 0_{N-2}} W_{n_{2} n_{1} 0_{N-2} ; 0_{N-2} n_{2} n_{1}}^{+}, n_{1}+n_{2}=1 \\
& \lambda_{n_{1} n_{2} ; n_{1} n_{2}}^{(0)}=\left|W_{0_{N-2} n_{1} n_{2} ; n_{1} n_{2} 0_{N-2}}\right|^{2}, \quad n_{1}+n_{2}=1 \\
& \lambda_{11 ; 11}^{(0)}=\left|W_{0_{N-2} 11 ; 110_{N-2}}\right|^{2}
\end{aligned}
$$

\section{Obstacle for complete structural restoring}

However, the direct analysis of the operator $W$ shows that the following subsystem of system (45), (46)

$$
\begin{aligned}
& W_{0_{N-2} n_{1} n_{2} ; n_{2} n_{1} 0_{N-2}}=0 \\
& W_{J_{N-4} 00 n_{1} n_{2} ; 110_{N-2}}=0, \quad\left|J_{N-4}\right|=1,
\end{aligned}
$$

where $n_{1}+n_{2}=1$, is not solvable in general. In fact, this subsystem consists of $2+2(N-4)$ complex equations. According to (19), all the elements of $W$ appearing in (54) include the elements $\left(V_{0}\right)_{00 n_{1} n_{2} ; i_{1} i_{2} i_{3} i_{4}}$ of the matrix $V_{0}$ which introduces $\varphi$-parameters. The direct analysis of $V_{0}$ satisfying commutation condition (20) shows that the above elements of $V_{0}$ depend on the following set of ten $\varphi$-parameters:

$$
\varphi_{2,3}^{(k)}, \varphi_{2,5}^{(k)}, \varphi_{2,8}^{(k)}, \varphi_{3,5}^{(k)}, \varphi_{3,8}^{(k)}, \quad k=1,2
$$

Therefore, system (54) can not be solved even for the shortest chain of $N=6$ nodes (2-qubit sender and 4-qubit extended receiver), when the number of complex equations in (54) is six. Therefore, solvability of (54) requires increasing the dimensionality of the extended receiver. In other words, the dimensionality of the extended receiver required for the complete struc-

tural restoring of the diagonal part of the zero-order coherence matrix increases with the length $N$ of the communication line. Such restoring is not considered in our paper. 


\section{Structural restoring of non-diagonal elements}

The obstacle pointed out in Sec IIE1 doesn’t arise in restoring the nondiagonal part of the zero-order coherence matrix. Eqs.(31) and (42) providing such restoring read $\left(n_{1}+n_{2}=\right.$ 1):

$$
\begin{aligned}
& W_{0_{N-2} n_{1} n_{2} ; n_{2} n_{1} 0_{N-2}}=0, \\
& \sum_{\left|J_{N-2}\right|=1} W_{J_{N-2} n_{1} n_{2} ; 110_{N-2}} W_{110_{N-2} ; J_{N-1} n_{2} n_{1}}^{+}=0 .
\end{aligned}
$$

As the result, the elements of the partially restored zero-order coherence matrix (Eqs. (39), (35) and (40)) read $\left(n_{1}+n_{2}=1\right)$ :

$$
\begin{aligned}
& \rho_{n_{1} n_{2} ; n_{2} n_{1}}^{(R ; 0)}=\lambda_{n_{1} n_{2} ; n_{2} n_{1}}^{(0)} \rho_{n_{1} n_{2} ; n_{2} n_{1}}^{(S ; 0)}, \\
& \rho_{n_{1} n_{2} ; n_{1} n_{2}}^{(R ; 0)}=\tilde{\lambda}_{n_{1} n_{2} ; 11}^{(0)} \rho_{11 ; 11}^{(S ; 0)}+\lambda_{n_{1} n_{2} ; n_{1} n_{2}}^{(0)} \rho_{n_{1} n_{2} ; n_{1} n_{2}}^{(S ; 0)}, \\
& \rho_{11 ; 11}^{(R ; 0)}=\lambda_{11 ; 11}^{(0)} \rho_{11 ; 11}^{(S ; 0)}, \\
& \rho_{00 ; 00}^{(R ; 0)}=\rho_{00 ; 00}^{(S ; 0)}-\sum_{i_{1}+i_{2}=1} \lambda_{i_{1} i_{2} ; i_{1} i_{2}}^{(0)} \rho_{i_{1} i_{2} ; i_{1} i_{2}}^{(S ; 0)},-\left(\lambda_{11 ; 11}^{(0)}+\tilde{\lambda}_{01 ; 11}^{(0)}+\tilde{\lambda}_{10 ; 11}^{(0)}\right) \rho_{11 ; 11}^{(S ; 0)},
\end{aligned}
$$

where $\lambda_{i j}^{(0)}$ are given in eqs.(511) - (153) and

$$
\tilde{\lambda}_{n_{1} n_{2} ; 11}^{(0)}=\sum_{\left|J_{N-2}\right|=1}\left|W_{J_{N-2} n_{1} n_{2} ; 110_{N-2}}\right|^{2} .
$$

\section{F. Results on structural restoring of non-diagonal part of $\rho^{(S)}$}

Now we collect the results of Secs IIICHIIE on structural restoring of the non-diagonal part of the initial sender's density matrix.

The constraints for the elements of $W$, which must be resolved for $\varphi$-parameters of the unitary transformation (24) and which provide the required restoring, are given by Eqs.(30), (31) (or (56)) and (57). For convenience, we collect them:

$$
\begin{aligned}
& \sum_{\left|J_{N-2}\right|=1} W_{J_{N-2} 00 ; m_{1} m_{2} 0_{N-2}} W_{110_{N-2} ; J_{N-2} n_{1} n_{2}}^{+}=0, m_{1}+m_{2}=n_{1}+n_{2}=1, \\
& W_{0_{N-2} n_{1} n_{2} ; n_{2} n_{1} 0_{N-2}}=0, \quad n_{1}+n_{2}=1, \\
& \sum_{\left|J_{N-2}\right|=1} W_{J_{N-2} n_{1} n_{2} ; 110_{N-2}} W_{110_{N-2} ; J_{N-2} n_{2} n_{1}}^{+}=0, \quad n_{1}+n_{2}=1 .
\end{aligned}
$$


This system consists of 7 complex equations for $42 \varphi$-parameters in the unitary transformation (24) and therefore can be satisfied, which is confirmed below in Sec.IV. We remark, that the number of the $\varphi$-parameters in the similar unitary transformation (24) associated with the three-qubit extended receiver is 12 , which is not enough to solve 7 complex equations (63). This fact justifies our choice of the unitary transformation on the four-qubit extended receiver as a minimal state-restoring tool. The restored elements are given by eqs.(25), (32),

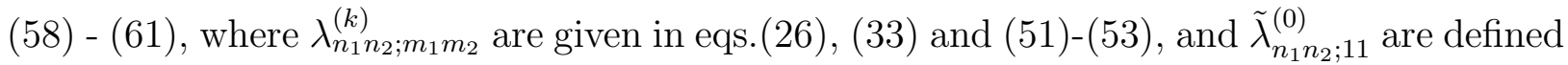
in (62). We notice that the number of equations in system (63) representing the system of constraint on $W$ does not depend on the length $N$ of a communication line. Therefore, if we disregard the structural restoring of the diagonal part of the zero-order coherence matrix, then the required dimensionality of the extended receiver does not depend on the length $N$ of the communication line as well.

\section{NUMERICAL PARTIAL STRUCTURAL RESTORING}

Now we numerically construct the partially structurally restored states discussed in Sec \IIF using the communication line of $N=42$ nodes. We show that this can be performed using the $\varphi$-parameters in the optimizing unitary transformation. In addition, we would like to maximize the scale factors corresponding to the restored elements, i.e., the factors $\left|\lambda_{00 ; 11}^{(2)}\right|,\left|\lambda_{n_{1} n_{2} ; m_{1} m_{2}}^{(1)}\right|$ and $\left|\lambda_{01 ; 10}^{(0)}\right|$ which can be considered as damping factors because all of them are less than one by absolute value. The reason of such damping is dispersion of a propagating state. Of course, we would prefer the minimal damping (i.e. all scale factors approach one by absolute value). However, it is natural that all of them can not reach their maximal values simultaneously. Therefore, we find such $\varphi$-parameters that maximize the absolute value of a selected scale factor. The solution of this problem is not unique, therefore we perform 1000 numerical experiments and choose the case corresponding to the maximal sum of absolute values of all other scale factors. This requirement fixes the optimizing unitary transformation. The results of such optimization are collected in Table I, where each of the 1st to 6th rows includes the scale factors corresponding to the maximization of, respectively, $\left|\lambda_{01 ; 10}^{(0)}\right|,\left|\lambda_{00 ; 01}^{(1)}\right|,\left|\lambda_{00 ; 10}^{(1)}\right|,\left|\lambda_{01 ; 11}^{(1)}\right|,\left|\lambda_{10 ; 11}^{(1)}\right|$ and $\left|\lambda_{00 ; 11}^{(2)}\right|$. The 7 th row in this table corresponds to the maximization of the sum of all above scale factors. Again, we perform 1000 numerical experiments and choose the case corresponding to the maximal value of the 
minimal scale factor. We see that the scale factors shown in the 7 th row are very close to those shown in the first row corresponding to the optimization of the scale factor $\lambda_{01 ; 10}^{(0)}$. Moreover, the absolute values of all scale factors are valuable and the most uniform in these two rows.

As an example we represent the set of $\varphi$-parameters for the optimizing unitary transformation (24) corresponding to the 7 th row of Table I;

$$
\begin{aligned}
& \varphi_{2,3}^{(1)}=3.2173, \quad \varphi_{2,3}^{(2)}=4.6606, \quad \varphi_{6,7}^{(1)}=3.1337, \quad \varphi_{6,7}^{(2)}=3.2409, \\
& \varphi_{2,5}^{(1)}=1.5820, \quad \varphi_{2,5}^{(2)}=4.1773, \quad \varphi_{6,9}^{(1)}=0.4114, \quad \varphi_{6,9}^{(2)}=5.7006, \\
& \varphi_{2,8}^{(1)}=1.9863, \quad \varphi_{2,8}^{(2)}=3.8653, \quad \varphi_{6,10}^{(1)}=1.7915, \quad \varphi_{6,10}^{(2)}=4.5986 \text {, } \\
& \varphi_{3,5}^{(1)}=2.9836, \quad \varphi_{3,5}^{(2)}=2.8152, \quad \varphi_{6,11}^{(1)}=2.8966, \quad \varphi_{6,11}^{(2)}=0.1255 \text {, } \\
& \varphi_{3,8}^{(1)}=1.6892, \quad \varphi_{3,8}^{(2)}=1.5472, \quad \varphi_{7,9}^{(1)}=5.0837, \quad \varphi_{7,9}^{(2)}=2.4180 \text {, } \\
& \varphi_{4,6}^{(1)}=-0.0758, \varphi_{4,6}^{(2)}=5.9730, \quad \varphi_{7,10}^{(1)}=0.6389, \quad \varphi_{7,10}^{(2)}=5.6101 \text {, } \\
& \varphi_{4,7}^{(1)}=2.9802, \quad \varphi_{4,7}^{(2)}=6.5792, \quad \varphi_{7,11}^{(1)}=3.7066, \quad \varphi_{7,11}^{(2)}=3.2203 \text {, } \\
& \varphi_{4,9}^{(1)}=3.3090, \quad \varphi_{4,9}^{(2)}=3.4037, \quad \varphi_{9,10}^{(1)}=2.7775, \quad \varphi_{9,10}^{(2)}=5.5717 \text {, } \\
& \varphi_{4,10}^{(1)}=1.9777, \quad \varphi_{4,10}^{(2)}=2.0048, \quad \varphi_{9,11}^{(1)}=0.5211, \quad \varphi_{9,11}^{(2)}=6.2622 \text {, } \\
& \varphi_{4,11}^{(1)}=1.5586, \quad \varphi_{4,11}^{(2)}=4.5361, \quad \varphi_{10,11}^{(1)}=6.3862, \varphi_{10,11}^{(2)}=4.2717 \text {. } \\
& \varphi_{5,8}^{(1)}=2.5019, \quad \varphi_{5,8}^{(2)}=2.9224 \text {, }
\end{aligned}
$$

Implementing this optimizing transformation we perform mapping of an arbitrary nondiagonal elements of the density matrix $\rho^{(S)}(0)$ into the appropriate elements of the receiver's density matrix $\rho^{(R)}$ with the corresponding damping factors collected in the 7 th row.

We notice also that the maximization of $\left|\lambda_{00 ; 01}^{(1)}\right|,\left|\lambda_{00 ; 10}^{(1)}\right|$ and $\left|\lambda_{00 ; 11}^{(2)}\right|$ (see respectively the 2nd, 3rd and 6st rows in Table I) makes these factors largest of all others. In other cases the maximized scale factor is not the largest one, see the 1st, 4th and 5th rows in this table.

\section{CONCLUSIONS}

A variant of remote state creation is the scaled state creation which is a simple well described map of the initial sender's state into the receiver's one. In the ideal case this map reduces the state creation to the multiplication of each matrix element (except the diagonal element providing normalization) by a scalar scale factor and therefore can be regarded as a consequent development of the ideal state transfer protocol. We refer to such map as a structural restoring of the initial sender's state. 


\begin{tabular}{|cccccc|}
\hline$\lambda_{01 ; 10}^{(0)}$ & $\lambda_{00 ; 01}^{(1)}$ & $\lambda_{00 ; 10}^{(1)}$ & $\lambda_{01 ; 11}^{(1)}$ & $\lambda_{10 ; 11}^{(1)}$ & $\lambda_{00 ; 11}^{(2)}$ \\
\hline $\mathbf{0 . 3 5 0 1} e^{2.6200 i}$ & $0.3871 e^{0.5993 i}$ & $0.9046 e^{-3.0639 i}$ & $0.2138 e^{-1.7284 i}$ & $0.4996 e^{1.9348 i}$ & $0.5522 e^{-1.1291 i}$ \\
$0.0044 e^{-2.6047 i}$ & $\mathbf{0 . 8 1 2 2} e^{0.2813 i}$ & $0.0055 e^{-2.3234 i}$ & $0.0948 e^{2.3781 i}$ & $0.0006 e^{-1.3004 i}$ & $0.1167 e^{2.6594 i}$ \\
$0.0780 e^{2.0820 i}$ & $0.0833 e^{1.1028 i}$ & $\mathbf{0 . 9 3 5 9} e^{-3.0983 i}$ & $0.0066 e^{-1.0999 i}$ & $0.0744 e^{3.1013 i}$ & $0.0794 e^{0.0029 i}$ \\
$0.0130 e^{1.2029 i}$ & $0.7883 e^{-2.7766 i}$ & $0.0165 e^{-1.5737 i}$ & $\mathbf{0 . 5 5 2 5} e^{-0.1457 i}$ & $0.0116 e^{-1.3486 i}$ & $0.7010 e^{-2.9223 i}$ \\
$0.0557 e^{3.0015 i}$ & $0.0622 e^{0.0781 i}$ & $0.8953 e^{3.0796 i}$ & $0.0387 e^{1.5415 i}$ & $\mathbf{0 . 5 5 6 8} e^{-1.4599 i}$ & $0.6219 e^{1.6197 i}$ \\
$0.0092 e^{-1.7244 i}$ & $0.2950 e^{2.5666 i}$ & $0.0312 e^{0.8422 i}$ & $0.2136 e^{-0.7733 i}$ & $0.0226 e^{0.9510 i}$ & $\mathbf{0 . 7 2 3 9} e^{1.7932 i}$ \\
$0.3489 e^{-1.7503 i}$ & $0.3868 e^{0.1608 i}$ & $0.9019 e^{-1.5895 i}$ & $0.2201 e^{0.2186 i}$ & $0.5132 e^{1.9689 i}$ & $0.5690 e^{0.3794 i}$
\end{tabular}

TABLE I: Optimized scale factors for the partial structural restoring of the two-qubit state in the communication line of $N=42$ nodes. The rows from 1 to 7 correspond to the maximization of, respectively, $\left|\lambda_{01 ; 10}^{(0)}\right|,\left|\lambda_{00 ; 01}^{(1)}\right|,\left|\lambda_{00 ; 10}^{(1)}\right|,\left|\lambda_{01 ; 11}^{(1)}\right|,\left|\lambda_{10 ; 11}^{(1)}\right|,\left|\lambda_{00 ; 11}^{(2)}\right|$ and the sum of the absolute values of all scale factors (the amplitudes of the maximized scale factors are in bold). In each case (except the last one) we also maximize the sum of the absolute values of all other scale factors.

In our case of the partial structural restoring of the two-qubit density matrix the above scaling is implemented only for the non-diagonal elements, while the diagonal elements remain uncontrolled. In addition, these scales are less then one by absolute value due to dispersion of a propagating state. This prompts us to call them the damping factors. In the proposed protocol we take into account the independent evolution of the MQ-coherence matrices. The basic restoring tool is the unitary transformation of the so-called extended receiver consisting of four last nodes of the communication line and embedding the receiver itself. We show that the facility of restoring the non-diagonal part is independent on the total length of the communication line, unlike the diagonal part, whose restoring requires the larger number of optimizing parameters in the unitary transformation and this number increases with $N$. The diagonal part restoring is postponed for further study.

We remark that the protocol of the block-scaled state transfer proposed in Ref. [20] doesn't involve the optimizing unitary transformation of the extended receiver. Those states have the same scale factor $\lambda^{(k)}$ for all the elements inside of each $\pm k$-order coherence matrix (up to the normalization of the zero-order coherence matrix). In addition, each block 
$\rho^{(S ;-1)}+\rho^{(S ; 1)}$ and $\rho^{(S ;-2)}+\rho^{(S ; 2)}$ carry only one arbitrary scalar parameter, while the parameter of the zero-order coherence matrix is fixed by the requirement of maximizing the state-subspace covered by the parameters of the nonzero-order coherence matrices. In our paper, the scale factors for all elements are independent from each other and the first- and second-order coherence matrices carry, respectively, four and one complex parameters (remember that the zero-order coherence matrix is uncontrolled in our case). This enhances the capability of our protocol as an information transfer tool, while additional relations among the scale factors might cause appropriate relations among the elements of $\rho^{(S)}(0)$ (which are completely independent in our case) and thus reduce the encoded information. Nevertheless, studying possible relations among the scale factors is a meaningful problem which deserves the further study.

Finally, we notice that the optimizing unitary transformation used in the restoring protocol can be considered as a tool for solving the linear algebraic systems because it reduces the linear combinations of the elements of $\rho^{(S)}$ to the appropriate elements multiplied by the damping factors. The problem of reducing this damping appears to be of a principal meaning.

This work is partially supported by the program of the Presidium of RAS No. 5 "Electron resonance, spin-dependent electron effects and spin technology" and by the Russian Foundation for Basic Research (Grant No.16-03-00056).

[1] S. Bose, Phys. Rev. Lett. 91, 207901 (2003)

[2] M.Christandl, N.Datta, A.Ekert, and A.J.Landahl, Phys.Rev.Lett. 92, 187902 (2004)

[3] C.Albanese, M.Christandl, N.Datta, and A.Ekert, Phys.Rev.Lett. 93, 230502 (2004)

[4] P.Karbach and J.Stolze, Phys.Rev.A 72, 030301(R) (2005)

[5] G.Gualdi, V.Kostak, I.Marzoli, and P.Tombesi, Phys.Rev. A 78, 022325 (2008)

[6] A.Wójcik, T.Luczak, P.Kurzyński, A.Grudka, T.Gdala, and M.Bednarska Phys. Rev. A 72, $034303(2005)$

[7] L. Banchi, T. J. G. Apollaro, A. Cuccoli, R. Vaia, and P. Verrucchi, Phys.Rev.A 82, 052321 (2010)

[8] A. Zwick and O. Osenda, J. Phys. A: Math. Theor. 44, (2011) 105302. 
[9] L. Banchi, T. J. G. Apollaro, A. Cuccoli, R. Vaia and P. Verrucchi, New J. Phys. 13, 123006 (2011)

[10] T. J. G. Apollaro, L. Banchi, A. Cuccoli, R. Vaia, and P. Verrucchi, Phys. Rev. A 85 (2012), 052319

[11] J.Stolze, G. A. Álvarez, O. Osenda, and A. Zwick in Quantum State Transfer and Network Engineering. Quantum Science and Technology, ed. by G.M.Nikolopoulos and I.Jex, Springer Berlin Heidelberg, Berlin, p.149 (2014)

[12] N.A.Peters, J.T.Barreiro, M.E.Goggin, T.-C.Wei, and P.G.Kwiat, Phys.Rev.Lett. 94, 150502 (2005)

[13] N.A.Peters, J.T.Barreiro, M.E.Goggin, T.-C.Wei, and P.G.Kwiat in Quantum Communications and Quantum Imaging III, ed. R.E.Meyers, Ya.Shih, Proc. of SPIE 5893 (SPIE, Bellingham, WA, 2005)

[14] G.Y. Xiang, J.Li, B.Yu, and G.C.Guo Phys. Rev. A 72, 012315 (2005)

[15] A.I.Zenchuk, Phys. Rev. A 90, 052302(13) (2014)

[16] G. A. Bochkin and A. I. Zenchuk, Phys.Rev.A 91, 062326(11) (2015)

[17] R. F. Werner, Phys. Rev. A 40, 4277 (1989)

[18] J.Stolze and A.I.Zenchuk, Quant. Inf. Proc. 15, (2016) 3347

[19] E.B.Fel'dman, A.I.Zenchuk, JETP 125, 1042 (2017)

[20] G.A.Bochkin, E.B.Fel'dman, A.I.Zenchuk, Quant.Inf.Proc. 17, 218 (2018)

[21] G. Bochkin and A. Zenchuk, Qunt. Inf. Comp. 16, 1349 (2016)

[22] A.I.Zenchuk, J. Phys. A: Math. Theor. 45115306 (2012)

[23] A. W. Harrow, A. Hassidim, and S. Lloyd, Phys.Rev.Lett. 103, 150502 (2009)

[24] E. B. Fel'dman, S. Lacelle, Chem. Phys. Lett. 253, 27 (1996) 